DOI: $10.15593 / 2224-9982 / 2021.66 .08$

УДК 681.5

\author{
П.В. Петров ${ }^{1}$, В.А. Целищев ${ }^{1}$, Д.А. Кудерко \\ ${ }^{1}$ Уфимский государственный авиационный технический университет, Уфра, Россия \\ ${ }^{2} \mathrm{AO}$ «Технодинамика», Москва, Россия

\section{СОВЕРШЕНСТВОВАНИЕ ЧИСЛЕННЫХ МЕТОДОВ РАСЧЕТА ГИДРОМЕХАНИЧЕСКИХ УСТРОЙСТВ АВТОМАТИКИ ЛЕТАТЕЛЬНЫХ АППАРАТОВ}

\begin{abstract}
Посвящена рассмотрению основных проблем математического моделирования и наиболее рационального представления результатов численного исследования гидромеханических регуляторов летательных аппаратов на стадии практического проектирования.

Традиционно анализ и синтез устройств гидроавтоматики авиационных систем выполняются на основе линейных частотных методов теории автоматического регулирования, которые позволяют получать приближенные характеристики: точность, устойчивость, управляемость. Основной задачей в этом случае становится выработка таких допущений и решений, которые позволили бы решить задачу в аналитической форме. Однако число нелинейных задач, доведенных до конца аналитическими методами, пренебрежимо мало. В такой ситуации компьютерное моделирование становится безальтернативным.

Механизация и частичная автоматизация вычислительного эксперимента привели к качественным изменениям результатов моделирования и позволили получить обобщенные диаграммы динамического состояния исследуемых систем.

На конкретном примере рассматриваются вопросы алгоритмического моделирования, анализа и численного исследования динамических характеристик системы автоматического управления летательных аппаратов на переходных режимах.

Наглядно продемонстрированно, как применение метода обобщенных переменных помогает представить результаты численных экспериментов в рациональном виде и определить влияние параметрических комплексов на основные характеристики, а также построить обобщенные динамические характеристики по основным критериям качества.

Ключевые слова: вычислительный эксперимент, система управления, летательный аппарат, диагностическое моделирование, нелинейные явления, устройства гидроавтоматики, математические модели, численное исследование, отказы, методика диагностирования.
\end{abstract}

\author{
P.V. Petrov ${ }^{1}$, V.A. Tselischev ${ }^{1}$, D.A. Kuderko ${ }^{2}$ \\ ${ }^{1}$ Ufa State Aviation Technical University, Ufa, Russian Federation \\ 2JSC "Technodinamika”, Moscow, Russian Federation
}

\title{
IMPROVEMENT OF NUMERICAL METHODS FOR CALCULATING HYDRO-MECHANICAL DEVICES OF AIRCRAFT AUTOMATION
}

\footnotetext{
The article is devoted to the consideration of the main problems of mathematical modeling and the most rational presentation of the results of numerical research of hydro-mechanical regulators of aircraft at the stage of practical design.

Traditionally, the analysis and synthesis of hydraulic automation devices of aviation systems are performed on the basis of linear frequency methods of the theory of automatic control, which allow us to obtain approximate characteristics: accuracy, stability, controllability. The main task in this case is to develop such assumptions and solutions that would allow solving the problem in an analytical form. However, the number of nonlinear problems completed by analytical methods is negligible. In such a situation, computer modeling becomes an alternative.

Mechanization and partial automation of the computational experiment led to qualitative changes in the simulation results and allowed us to obtain generalized diagrams of the dynamic state of the studied systems.

Using a specific example, the issues of algorithmic modeling, analysis and numerical study of the dynamic characteristics of the automatic control system of aircraft on transient modes are considered.

The paper clearly demonstrates how the application of the generalized variables method helps to present the results of numerical experiments in a rational form and to determine the influence of parametric complexes on the main characteristics and to construct generalized dynamic characteristics according to the main quality criteria

Keywords: computational experiment, control system, aircraft, diagnostic modeling, nonlinear phenomena, hydraulic automation devices, mathematical models, numerical research, failures, diagnostic methods.
} 


\section{Введение}

Процесс проектирования современных систем автоматики в первую очередь сопровождается усложнением математического аппарата, так как анализ и расчет таких систем приходится вести по нелинейным дифференциальным уравнениям [1].

К сожалению, дифференциальные уравнения в нелинейной постановке, как правило, не поддаются точному аналитическому решению. Приближенные аналитические методы (фазовые траектории и плоскости, припасовывание и др.) реализуются за счет существенных упрощений, имеют очень узкую сферу применения и, как правило, используются для описания систем небольших порядков [2].

Широкое распространение линейных методов анализа устройств гидроавтоматики связано в первую очередь со сложностью, а иногда и невозможностью решения нелинейных уравнений (и их систем) качественно и количественно (в некотором диапазоне), с достаточной точностью. А использование численного метода решения упиралось в уровень развития вычислительных средств и его программного обеспечения [3].

Развитие методов компьютерной математики существенно изменило ситуацию в этом вопросе [4]. Если раньше программирование было уделом продвинутых программистов, то теперь многие сложные задачи решаются сравнительно неопытными пользователями.

При сегодняшнем уровне развития вычислительных технологий $\mathrm{y}$ исследователя есть возможность отказаться от некоторых допущений и проводить исследования на более адекватных математических моделях, но эта возможность в настоящее время используется редко. Ввиду этого, во-первых, как уже говорилось, невозможно создать полную математическую модель или же эта модель настолько сложна, что найти решение по ней практически невозможно. Во-вторых, для полной модели требуется большой спектр необходимой информации, которая не всегда доступна и большей частью специфична (у каждого отдельного элемента есть свои особенности - физические, химические свойства, конструктивные особенности), поэтому приходится преобразовывать найденную информацию в удобный для конкретной модели вид. И в-третьих, наиболее труднопреодолимая проблема - многообразие и разобщенность методик расчетов объектов и дальнейшего математического моделирования. Но это направление со временем должно стать широко используемым методом.

Решение последних двух проблем, т.е. накопление информации и методов ее преобразования из одного вида в другой, а также разработка универсальных методов расчета, моделирования с последующим анализом, позволит избавить от проблем как проектировщика (от изменения вида представления модели), так и программиста (от изучения сущности каждого конкретного агрегата).

Особенно существенный прогресс в этом вопросе имеет место у прикладного программного обеспечения [5]. Здесь помимо роста числа стандартных программ наблюдается знаменательное качественное изменение. К услугам разработчика имеются готовые библиотеки научно-технических программ, электронные справочники и так далее и, прежде всего, пакеты прикладных программ - важнейший инструмент выполнения типовых расчетов.

Компьютерное моделирование в ходе многолетнего развития позволяет создавать информационное поле, включающее все потенциально полезные модели и оперативное их использование [6].

Прежде чем приступать к компьютерному моделированию, необходимо определиться с постановкой задачи и целями, которые мы должны достигнуть в результате моделирования исследуемой системы. Большое разнообразие гидромеханических систем обусловливает разнообразие моделей, применяемых при выборе базовых параметров [7]. Разомкнутый и следящий гидроприводы, системы управления и регулирования летательного аппарата (ЛА) или двигателя, приводы станка или транспортной машины и прочие гидросистемы требуют индивидуального подхода. К примеру, выбор параметров системы стабилизации режима авиационного двигателя должен выполняться с целью достижения заданной точности, т.е. в данном случае система приемистости двигателя должна обеспечить заданную траекторию 
разгона [8]. Другой пример: гидропривод системы управления летательного аппарата помимо безусловного преодоления статических нагрузок и скоростей должен отвечать определенным динамическим требованиям или в станочном гидроприводе большое значение уделяется точности и плавности движения и т.п. Такое разнообразие существенно затрудняет выработку общих рекомендаций моделирования систем. Приходится ограничиваться некоторыми рекомендациями по использованию статистических соображений и прикидочных расчетов [9].

Несколько иначе выглядит задача моделирования отдельных устройств [10]. С одной стороны, ситуация является более простой, число требований значительно меньше и назначение приоритетного требования более определенно. С другой - требуется выбрать большее число более конкретизированных базовых параметров (проходные сечения дросселирующих устройств, жесткости пружин, площади гидроцилиндров и т.п.). Для простых устройств имеется возможность, по крайней мере, некоторой механизации процесса моделирования. Имеются многочисленные подходы, среди которых наиболее успешным, несомненно, является метод типовых динамических звеньев, позволяющий генерировать практически любую, но непременно линейную структуру. В этом методе принимается во внимание только динамическое поведение системы. Передаточные функции показывают, как звено передает динамический сигнал. При этом совершенно неважно, из чего устроено это звено, главное - то, что оно делает. Полу- чается весьма наглядный и универсальный инструмент анализа динамики. Как же в данном случае рассматривать устройства, имеющие более сложную структуру, построенную на различной элементной базе? Основы компьютерного моделирования подобных систем в нелинейной постановке на данный момент практически отсутствуют.

\section{Пример численного исследования и обобщенного анализа работы САР на переходных режимах}

На рис. 1 приведена принципиальная схема гидромеханического регулятора, который обеспечивает работу как установившихся статических режимов, так и переходных (разгон и дросселирование), т.е. является всережимным [11].

Управление двигателя на режимах приемистости рассматривается на примере одновального газотурбинного двигателя (ГТД) с постоянной геометрией газовоздушного тракта. Известно, что для разгона ротора необходимо иметь избыточный момент на турбине, по сравнению с моментом на стационарных режимах. В рассматриваемом случае это приводит к необходимости создания избытков топлива.

Рассмотрим работу изодромного регулятора частоты вращения при больших возмущениях - более $5 \%$ (регулирование переходных режимов «разгон» и «дросселирование»). При таких больших возмущениях, например, при приемистости, т.е. при переводе ручки управления двигателя с малого газа на максимальный режим за короткое время или мгновенно.

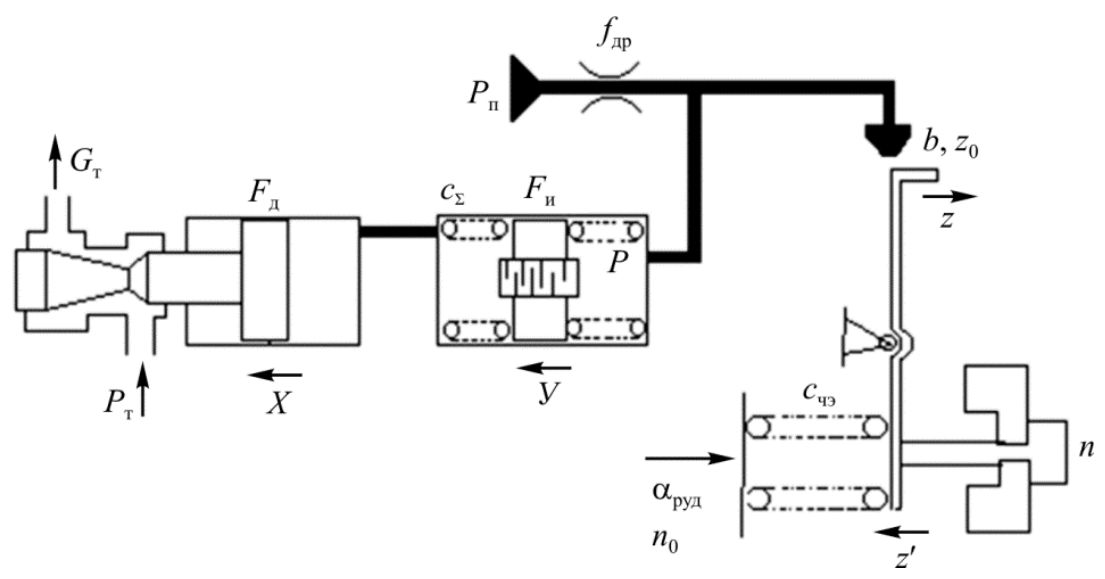

Рис. 1. Схема изодромного регулятора 
Математическая модель автомата разгона [12] в этом случае должна быть дополнена следующими функциями пользователя, которые ограничивают, к примеру, перемещение поршня изодрома, скорость поршня изодрома, а также не дают значениям геометрии щели распределителя становиться отрицательными и т.п.

Некоторые задачи, являющиеся частным случаем общей задачи, уже решены аналитически. Так, в случае малых возмущений (меньше двух-трех процентов) дифференциальные уравнения могут быть существенно упрощены путем их линеаризации. В целом эту систему уравнений можно свести к одному уравнению. Однако особый интерес представляет общая задача, в которой: 1) приходится решать несколько уравнений одновременно; 2) нельзя пренебречь ни одним членом уравнений; 3) в уравнениях имеются нелинейности, которые при конечных возмущениях невозможно линеаризовать; 4) уравнения являются совместными. Так, частота вращения в первом уравнении является искомой величиной, а во втором и четвертом выступает в роли параметра и т.д.

Любого из перечисленных свойств достаточно, чтобы отказаться от аналитического решения.

Модель системы регулирования в самом простом варианте включает в себя по крайней мере еще одно уравнение для регулятора. Если положить, что поршень гидроцилиндра невесомый и перепад давления на нем отсутствует, то модель регулятора может быть получена из следующих соотношений:

$$
\begin{aligned}
& F \frac{d z}{d t}=K_{Q x} x_{\text {щ }} ; \\
& x_{\text {щ }}=K_{\mathrm{p}} y-K_{\text {ос }} z ; \\
& y=K_{\text {чэ }} n ; \\
& G=K_{\text {ди }} z,
\end{aligned}
$$

где $F$ - площадь поршня гидроцилиндра; $z$ перемещение дозирующей иглы; $K_{Q x}-$ коэффициент усиления по расходу; $x_{\text {щ }}$ - ширина щели золотника; $K_{\mathrm{p}}$ - коэффициент усиления рычага; $y$ - перемещение чувствительного элемента; $K_{\mathrm{oc}}$ - коэффициент обратной связи; $K_{\text {чэ }}-$ коэффициент усиления чувствительного элемента; $G$ - расход топлива; $K_{\text {ди }}-$ коэффициент усиления дозирующей иглы.
Первые три уравнения дают известное выражение для следящего гидроусилителя:

$$
T_{\text {гу }} \frac{d z}{d t}+z=K_{\text {гу }} \Delta n,
$$

где $T_{\text {гу }}, K_{\text {гу }}$ - постоянная времени и коэффициент усиления гидроусилителя, $T_{\text {гу }}=\frac{F}{K_{Q x} K_{\text {ос }}}, K_{\text {гу }}=\frac{K_{\text {чэ }} K_{\mathrm{p}}}{K_{\text {ос }}}$.

Для случая торможения двигателя на величину $\Delta n_{0}$ правая часть выражения будет иметь вид $\Delta n=-\Delta n_{0}+n_{0}-n$, а для разгона $\Delta n=\Delta n_{0}-n_{0}+n$.

Для астатического регулятора в случае внешних воздействий соответствующая система имеет вид

$$
\begin{aligned}
& F_{\text {пор }} \frac{d z}{d t}=K_{Q x} x ; \\
& x=K_{\text {р }} y \\
& y=K_{\text {чэ }} \Delta n .
\end{aligned}
$$

В целом система уравнений может быть проинтегрирована даже простейшим методом Рунге - Кутты с фиксированным шагом, но большой разброс значений переменных приводит к необходимости назначения очень большого числа шагов и, как следствие, к существенным затратам машинного времени. Кроме того, имеется около двух десятков переменных, влияние которых следовало бы изучить. Для преодоления указанных затруднений приходится прибегать ко всяческим ухищрениям, например предварительно умножать каждый член второго уравнения на модуль упругости жидкости, делить каждый член третьего уравнения на площадь поршня дозирующей иглы и т.п.

Комплексная математическая модель САР совместно с моделью двигателя рассматривалась в предыдущих статьях. В частности, рассматривалась нелинейная модель двигателя с временным регулятором разгона [13]. В продолжение к этим статьям рассмотрим метод рационализации численных исследований. Суть его заключается в переходе от первоначальных размерных к безразмерным обобщенным переменным, что позволяет 
уменьшить число аргументов задачи и построить обобщенные характеристики исследуемой системы.

Большое количество переменных и разброс их числовых значений затрудняют решение и составление общих характеристик, поэтому основное исследование выполняется с использованием безразмерных переменных [14].

В качестве масштабов преобразования приняты следующие величины: постоянная времени двигателя на стационарном исходном режиме $T_{\text {дв }}$ - масштаб времени; давление $p_{0}$ в управляющей камере на стационарном режиме - масштаб давления; частота вращения $n_{0}$ на стационарном расчетном режиме - масштаб частоты вращения; максимальное перемещение статического поршня $y_{m}$, которое удобно определить как $y_{m}=\frac{p_{0} F_{u}}{c_{\Sigma}}$, т.е. перемещение при действии максимального перепада на статическом поршне.

Безразмерные переменные определяются как отношения размерных величин и соответствующих масштабов:

$$
\begin{gathered}
\bar{t}=\frac{t}{T_{\text {дв }}} ; \bar{n}=\frac{n}{n_{0}} ; \bar{p}=\frac{p}{p_{0}} ; \bar{z}=\frac{z}{y_{m}} ; \\
\bar{y}=\frac{y}{y_{m}} ; \bar{x}=\frac{x}{y_{m}} .
\end{gathered}
$$

После введения масштабов и несложных преобразований получена система безразмерных уравнений, принятых к систематическому исследованию:

$$
\left\{\begin{array}{l}
\sqrt{1-\Delta \bar{p}}=\left(1+\frac{y_{m}}{z_{0}}\left(-\Delta \bar{z}_{0}+\Delta \bar{z}\right)\right) \sqrt{1+\bar{p}}+ \\
+\bar{T}_{y} \frac{d \bar{y}}{d \bar{t}}+\bar{f}_{\Pi} \sqrt{\Delta \bar{p}}+\bar{T}_{v}(1+\Delta \bar{y}) \frac{d \bar{p}}{d \bar{t}} ; \\
\bar{f}_{\text {п }} \sqrt{\Delta \bar{p}}+\bar{T}_{y} \frac{d \bar{y}}{d \bar{t}}=\bar{T}_{y} \bar{F}_{\text {ди }} \frac{d \bar{x}}{d \bar{t}} ; \\
\bar{T}_{c}^{2} \frac{d^{2} \bar{y}}{d \bar{t}^{-2}}=\Delta \bar{p}-2 \bar{T}_{c} \frac{d \bar{y}}{d \bar{t}}+\Delta \bar{y} ; \\
\frac{d \bar{n}}{d \bar{t}}+\Delta \bar{n}=k_{\text {дв }}\left(\Delta \bar{G}_{T}+\Delta \bar{f}_{\text {в }}\right) ; \\
\Delta \bar{z}=\bar{k}_{n} \Delta \bar{n},
\end{array}\right\}
$$

где $T_{y}=\frac{F_{u} y_{m}}{Q_{0}}, T_{c}=\sqrt{\frac{m}{c_{\Sigma}}}, T_{v}=\frac{p_{0} V_{0}}{E Q_{0}}-$ соответственно постоянная времени гидроусилителя, период собственных колебаний поршня и постоянная времени наполнения; $\bar{T}_{y}=\frac{T_{y}}{T_{\text {дв }}}$, $\bar{T}_{c}=\frac{T_{c}}{T_{\text {дв }}}, \bar{T}_{v}=\frac{T_{v}}{T_{\text {дв }}}-$ соответствующие относительные значения времени, критерии гомохронности, а также безразмерные коэффициенты, входящие в эту систему уравнений, будут определятся по следующим формулам:

$$
\left(\begin{array}{l}
\bar{k}_{\text {дв }}=\frac{k_{\text {дв }} k_{\text {ди }} y_{m}}{n_{0}} ; \xi_{v}=\frac{k_{V}}{2 \sqrt{m c_{\Sigma}}} ; \\
\bar{k}_{\text {п }}=\frac{k_{\text {чэ }} \frac{l_{2}}{l_{1}} n_{0}}{y_{m}} ; \bar{f}_{\text {П }}=\frac{f_{\text {П }}}{f} ; \bar{F}_{\text {ди }}=\frac{F_{\text {ди }}}{F_{\text {И }}}
\end{array}\right) .
$$

Решение безразмерной системы ищется в виде зависимости безразмерных функций $\bar{n}, \bar{p}, \bar{y}, \bar{x}$ от безразмерного времени и комплексов, критериев подобия $\bar{t}, \bar{T}_{\text {г }}, \bar{T}_{\mathrm{m}}, \bar{T}_{v}, \bar{k}_{n}$, $\xi_{v}, \xi_{c}, \bar{f}_{\Pi}, \bar{f}_{1}, \bar{F}_{\text {ид }},{\overline{f_{01}}}_{\text {. }}$.

Протекание переходных процессов в автомате разгона зависит от семи безразмерных комплексов: $\bar{T}_{\mathrm{м}}, \bar{T}_{v}, \bar{k}_{n}, \bar{f}_{\Pi}, \bar{T}_{\Gamma}, \bar{F}_{\text {ид }}, \bar{f}_{1}$. Однако, как показали расчеты, первые три комплекса практически не влияют на характер переходных процессов. Относительная площадь дроссельного пакета изодрома подбирается из условия благоприятного протекания переходных процессов изодромного регулятора и для автомата разгона является заданной величиной. Ввиду этого синтез динамических характеристик временного автомата разгона следует осуществлять по трем последним комплексам [15].

На рис. 2 показано влияние $\bar{T}_{\text {гY }}$ на характер переходного процесса системы регулирования разгоном двигателя. Влияние постоянной времени $\bar{T}_{\Gamma}$ весьма существенно. $\mathrm{C}$ ее уменьшением увеличивается быстродействие всех этапов работы регулятора. Особенно сильное влияние оказывается на положение точки $D$, т.е. времени снятия поршня с 
упора. Очевидно, что, изменяя быстродействие гидроусилителя, можно обеспечить любое заданное быстродействие регулятора.

Наиболее реальным средством для достижения заданного протекания процессов автомата разгона являются относительная площадь поршней $\bar{F}_{\text {ид }}$ и площадь обводного пакета $f_{1}$.

На рис. 3 показано влияние $\bar{F}_{\text {ид }}$ на переходный процесс. В отличие от постоянной времени гидроусилителя, основное влияние этот параметр оказывает только на заключительную фазу процесса, начиная с точки $D$. Увеличивая относительную площадь поршней, можно добиться существенного роста быстродействия.

Увеличение площади обводного пакета также приводит к росту быстродействия, но влияние его не такое значительное (рис. 4).

На переходных процессах появляются характерные точки $C$ и $D$, соответствующие постановке и снятию с упора поршня изодрома.
Рассматриваемая в работе поэлементная модель двигателя является промежуточной. Уже сейчас ясно, что необходимо дальнейшее ее развитие, но не ожидается изменение структуры.

Совершенствование поэлементной модели САР связано с введением переменности коэффициентов полезного действия, использованием приведенных к внешним условиям параметров и характеристик, переходом к обобщенным параметрам, разработкой моделей двигателей других схем и пр.

На основе дальнейшего моделирования и анализа переходных процессов строятся обобщенные характеристики, к примеру график положения точки $D$ в зависимости от параметров $\overline{T_{\text {гY }}}, \bar{f}_{1}, \bar{F}_{\text {ид }}$ (рис. 5).

Количественную оценку быстродействия удобно осуществить по времени $\bar{T}_{D}$, которое определяется как время достижения поршнем изодрома точки $D$. На рис. 5 показаны

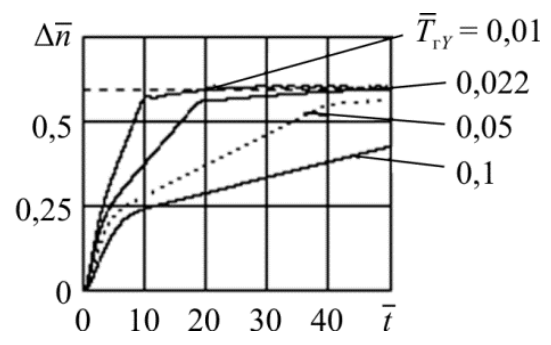

Рис. 2. Влияние изменения постоянной времени гидроусилителя на характер переходного процесса
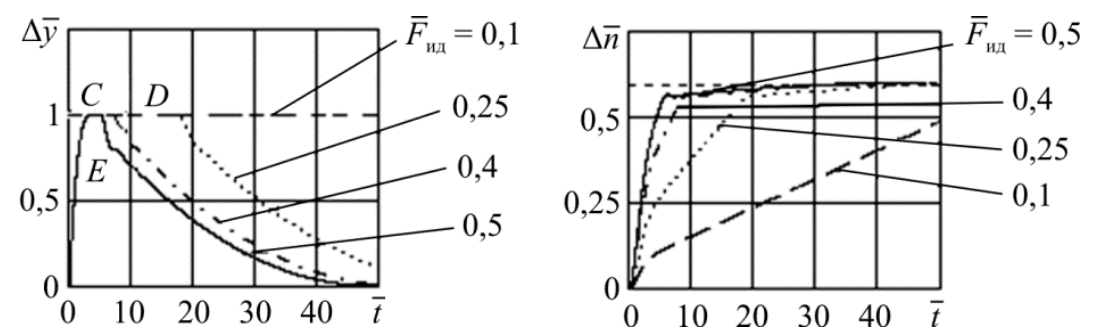

Рис. 3. Влияние относительной площади поршней
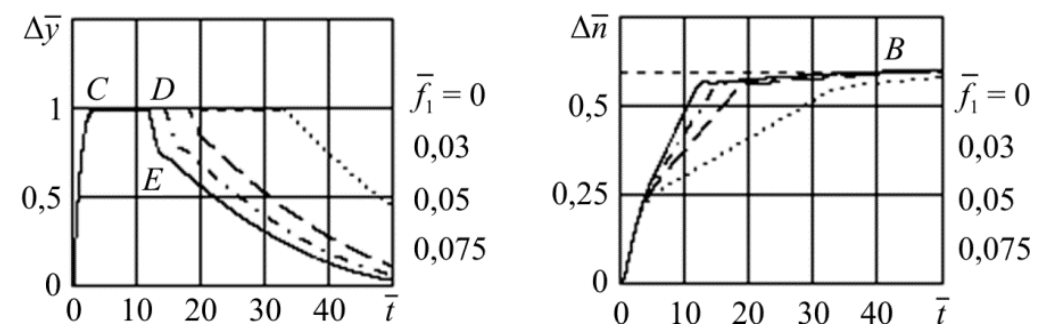

Рис. 4. Влияние площади обводного пакета на характер переходного процесса 


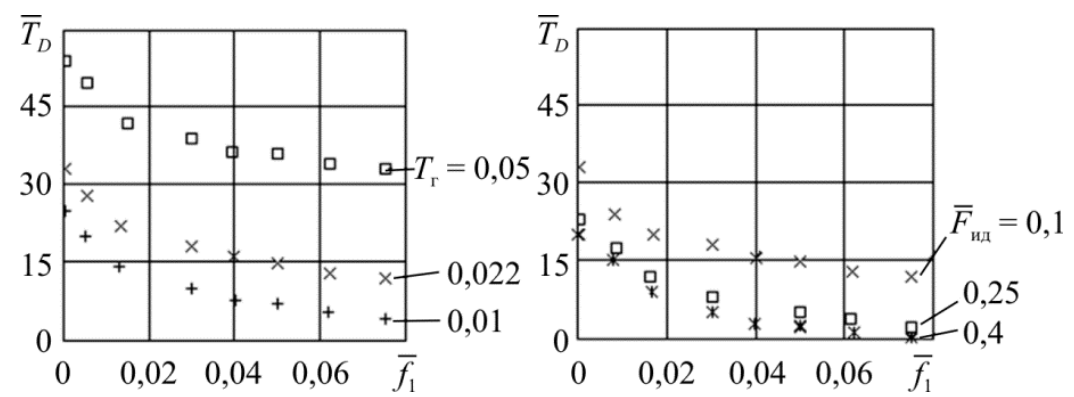

Рис. 5. Обобщенные характеристики быстродействия

результаты численного определения этого времени в зависимости от относительных значений площади поршней $\bar{F}_{\text {ид }}$, гидравлической постоянной времени и обводного дроссельного пакета $\bar{f}_{1}$. Как видно из рис. 5 , быстродействие увеличивается при увеличении значений площади и уменьшении гидравлической постоянной времени.

Для автомата разгона приоритетными являются требования к характеру переходного процесса. Точки переключения и характер протекания процессов между ними строго регламентируются в техническом задании.

\section{Заключение}

В статье рассматривается проблема проектирования энергоэффективных и надежных гидромеханических систем управления за счет разработки теоретико-экспериментальной методологии, инженерных и практических рекомендаций по интенсификации процесса разработки, проектирования и отработки основных гидромеханических узлов управления авиационным двигателем.

Поставленная цель достигается в результате следующих теоретических и эксперимен- тальных исследований. С учетом сделанного анализа определены дальнейшие направления исследований по регуляторам автоматики ГТД.

Описан свод рекомендаций по уменьшению или полному устранению вредного влияния различных особенностей физических процессов и дефектов на работу гидросистемы авиационного двигателя.

В работе реализованы новые принципы математического описания нелинейных гидромеханических устройств ДЛА и на их основе разработан комплекс нелинейных математических моделей, алгоритмов и программ, описывающих гидромеханические процессы, протекающие в устройствах гидроавтоматики, с учетом наиболее существенных нелинейных явлений.

Полученные результаты в виде набора алгоритмических моделей позволили оценить состояние гидроагрегатов при критических режимах, а также определить параметры, при которых они будут работать наиболее эффективно, т.е. появилась возможность предварительного параметрического синтеза системы, a в дальнейшем появится возможность диагностирования работы устройств гидроавтоматики на предконструкторском этапе.

\section{Библиографический список}

1. Петров П.В., Чернов Д.Д. Необходимость исследования нелинейных гидромеханических систем в обобщенных параметрах // Справочник. Инженерный журнал с приложением. - 2019. - № 4 (265). - С. 28-33

2. Петров П.В., Вакилова Л.Р. Сравнительная оценка вариантов возможных решений линейных и нелинейных задач гидромеханики с учетом результатов прогнозных исследований // Вестник Уфимского государственного авиационного технического университета. - 2020. - Т. 24, № 2 (88). - С. $79-87$.

3. Кривошеев И.А., Ахмедзянов Д.А., Кишалов А.Е. Имитационное моделирование работы авиационных ГТД с элементами систем управления // Вестник УГАТУ. Сер. Машиностроение. - 2008. № 2 (29). - C. 3-11.

4. Akhmedzyanov D.A., Kishalov A.E. Computer-aided design and construction development of the main elements of aviation engines // Lecture Notes in Electrical Engineering. - 2020. - Vol. 641. - P. 693-702.

5. Кишалов А.Е., Власова Е.С. Параметрическая идентификация математической модели ГТД в системе DVIGwp // Мавлютовские чтения: материалы Всерос. науч. конф. / Уфим. гос. авиац. ун-т. Уфа, 2007. - Т. 1. - С. 56-57. 
6. Ахмедзянов Д.А., Власова Е.С., Кишалов А.Е. Методология имитационного моделирования неустановившихся режимов работы авиационных ГТД // Вестник Самарского государственного аэрокосмического университета. - 2006. - № 2 (10). - С. 41-44.

7. Обзор и анализ параметров потока в основных узлах авиационных двигателей / Д.А. Ахмедзянов, А.Е. Кишалов, Ю.А. Шабельник, К.В. Маркина, Н.И. Полежаев // Молодежный вестник УГАТУ. 2012. - № 4 (5) - С. 25-36.

8. Ахмедзянов Д.А., Кишалов А.Е. Моделирование переходных процессов, протекающих при отладке автоматики при испытаниях ТРДДФ // Вестник Воронежского государственного технического университета. - 2011. - Т. 7, № 8. - С. 152-158.

9. Сунарчин Р.А. Выбор параметров гидромеханических регуляторов авиационных двигателей: учеб. пособие для студ. вузов, обучающихся по направлению 657400 «Гидравлическая, вакуумная и компрессорная техника», специальность 121100 «Гидравлические машины, гидроприводы и гидропневмоавтоматика» / Уфим. гос. авиац. техн. ун-т. - Уфа, 2005. - 87 с.

10. Петров П.В., Целищев В.А. Основы алгоритмического моделирования нелинейных гидромеханических устройств: учеб. пособие / Уфим. гос. авиац. техн. ун-т. - Уфа, 2012. - 137 с.

11. Сунарчин Р.А. Проектирование гидромеханических регуляторов авиационных двигателей: учеб. пособие для студ. всех форм обучения, обучающихся по направлению 657400 «Гидравлическая, вакуумная и компрессорная техника», специальность 121100 «Гидравлические машины, гидроприводы и гидропневмоавтоматика» / Уфим. гос. авиац. техн. ун-т. - Уфа, 2008. - 155 с.

12. Петров П.В., Целищев В.А. Численное моделирование работы систем автоматического управления авиационного газотурбинного двигателя на установившихся и переходных режимах // Вестник Пермского национального исследовательского политехнического университета. Аэрокосмическая техника. - 2019. - № 57. - С. 7-16.

13. Ахмедзянов Д.А., Кривошеев И.А., Сунарчин Р.А. Совместная работа авиационных газотурбинных двигателей и топливной автоматики на режимах разгона и торможения // Вестник СГАУ. - 2006. - № 1. - С. $24-25$.

14. Сунарчин Р.А., Машков М.А., Матросов А.В. Неустойчивость и автоколебания в гидравлических следящих приводах // Динамика и виброакустика. - 2018. - Т. 4, № 3. - С. 16-25.

15. Машков М.А., Матросов А.В., Сунарчин Р.А. Обобщенные характеристики электрогидравлического следящего привода // Неделя науки СПбПУ: материалы науч. форума с междунар. участием, г. СанктПетербург, 30 ноября - 05 декабря 2015 г. / Ин-т энергетики и трансп. сист. - СПб., 2015. - С. 91-93.

\section{References}

1. Petrov P.V., Chernov D.D. Neobkhodimost issledovaniya nelineynykh gidromekhanicheskikh sistem v obobshchennykh parametrakh [The necessity of studying nonlinear hydromechanical systems in generalized parameters]. Handbook. Engineering magazine with an appendix. 2019, no. 4 (265), pp. 28-33.

2. Petrov P.V., Vakilova L.R. Sravnitelnaya otsenka variantov vozmozhnykh resheniy lineynykh i nelineynykh zadach gidromekhaniki s uchetom rezul'tatov prognoznykh issledovaniy [Comparative evaluation of possible solutions to linear and nonlinear problems of hydromechanics, taking into account the results of predictive studies] (in Russian), Vestnik UGATU. 2020, vol. 24, no. 2 (88), pp. 79-87.

3. Krivosheev I.A., Akhmedzyanov D.A., Kishalov A.E. Imitatsionnoye modelirovaniye raboty aviatsionnykh GTD s elementami sistem upravleniya [Simulation modeling of the operation of aviation gas turbine engines with elements of control systems]. Vestnik UGATU. 2008, no. 2 (29) Ser. Mashinostroenie, p. 3-11.

4. Akhmedzyanov D.A., Kishalov A.E. Computer-Aided Design and Construction Development of the Main Elements of Aviation Engines. Lecture Notes in Electrical Engineering. 2020, vol. 641, pp. 693-702.

5. Kishalov A.E., Vlasov E.S. Parametricheskaya identifikatsiya matematiche-skoy modeli GTD v sisteme DVIGwp [Parametric identification of mathematical model of GTE in the system DVIGwp]. Mavlyutovskiye chteniya: materialy vseros. nauch. Conf. Ufa: UGATU, 2007, vol. 1, pp. 56-57.

6. Akhmedzyanov D.A., Vlasova E.S., Kishalov A.E. Metodologiya imitatsionnogo modelirovaniya neustanovivshikhsya rezhimov raboty aviatsionnykh GTD [Methodology of simulation modeling of unsteady modes of operation of aviation gas turbine engines]. Vestnik of the Samara State Aerospace University. 2006, no. 2 (10), pp. 41-44.

7. Akhmedzyanov D.A., Kishalov A.E., Shabelnik Yu.A., Markina K.V., Polezhaev N.I. Obzor i analiz parametrov potoka $\mathrm{v}$ osnovnykh uzlakh aviatsi-onnykh dvigateley [Review and analysis of flow parameters in the main nodes of aircraft engines]. Youth Bulletin of UGATU. 2012, no. 4 (5), pp. 25-36.

8. Akhmedzyanov D.A., Kishalov A.E. Modelirovaniye perekhodnykh protsessov, protekayushchikh pri otladke avtomatiki pri ispytaniyakh TRDDF [Modeling of transients occurring during automation debugging during turbofan tests]. Vestnik of the Voronezh State Technical University. 2011, vol. 7, no. 8, pp. 152-158. 
9. Sunarchin R.A. Vybor parametrov gidromekhanicheskikh regulyatorov aviatsionnykh dvigateley: ucheb. posobiye dlya studentov vuzov, obuchayushchikhsya po napravleniyu 657400 "Gidravl., vakuum. i kompressor. tekhnika" spetsial'nost' 121100 "Gidravl. mashiny, gidroprivody i gidropnevmoavtomatika"[The choice of parameters of hydro-mechanical regulators of aircraft engines: textbook. manual for university students studying in the direction 657400 "Hydraulic, vacuum. and the compressor. technique "specialty 121100" Hydraulic machines, hydraulic drives and hydropneumoautomatics"]. Ufa: Ufa State University. aviac. tech. un-t, 2005, 87 p.

10. Petrov P.V., Tselishchev V.A. Osnovy algoritmicheskogo modelirovaniya nelineynykh gidromekhanicheskikh ustroystv: ucheb. posobiye [Fundamentals of algorithmic modeling of nonlinear hydromechanical devices: textbook. Stipend]. Ufa: UGATU, 2012, 137 p.

11. Sunarchin R.A. Proyektirovaniye gidromekhanicheskikh regulyatorov aviatsionnykh dvigateley : uchebnoye posobiye dlya studentov vsekh form obucheniya, obuchayushchikhsya po napravleniyu 657400 "Gidravlicheskaya, vakuumnaya i kompressornaya tekhnika" spetsialnost 121100 "Gidravlicheskiye mashiny, gidroprivody i gidropnevmoavtomatika" [Design of hydro-mechanical regulators of aircraft engines : a textbook for students of all forms of education studying in the direction 657400 "Hydraulic, vacuum and compressor equipment" specialty 121100 "Hydraulic machines, hydraulic drives and hydropneumoautomatics"]. Federal Agency for Education, State Educational Institution of higher Prof. education Ufa State. Aviation Technical University-T.-Ufa: Ufa State University. aviation technical university, 2008, 155 p.

12. Petrov P.V. Chislennoye modelirovaniye raboty sistem avtomaticheskogo upravleniya aviatsionnogo gazoturbinnogo dvigatelya na ustanovivshikhsya i perekhodnykh rezhimakh [Numerical modeling of the operation of automatic control systems of an aviation gas turbine engine in steady-state and transient modes]. PNRPU Aerospace Engineering Bulletin. 2019, no. 57, pp. 7-16.

13. Akhmedzyanov D.A., Krivosheev I.A., Sunarchin R.A. Sovmestnaya rabota aviatsionnykh gazoturbinnykh dvigateley i toplivnoy avtomatiki na rezhimakh razgona i tormozheniya [Joint work of aviation gas turbine engines and fuel automation in acceleration and braking modes]. Vestnik of the SSAU, 2006, no. 1. pp. 24-25.

14. Sunarchin R.A., Mashkov M.A., Matrosov A.V. Neustoychivost i avtokolebaniya v gidravlicheskikh sledyashchikh privodakh [Instability and self-oscillation in hydraulic tracking drives]. Dynamics and vibroacoustics. 2018, vol. 4, no. 3, pp. 16-25.

15. Mashkov M.A., Matrosov A.V., Sunarchin R.A. Obobshchennyye kharakteristiki elektrogidravlicheskogo sledyashchego privoda [Generalized characteristics of an electrohydraulic tracking drive]. SPbPU Science Week: Materials of the scientific forum with international participation. Institute of Energy and Transport Systems, St. Petersburg, November 30-05, 2015. - St. Petersburg: Federal State Autonomous Educational Institution of Higher Education "Peter the Great St. Petersburg Polytechnic University", 2015, pp. 91-93.

\section{Об авторах}

Петров Павел Валерьевич (Уфа, Россия) - кандидат технических наук, доцент кафедры «Прикладная гидромеханика» ФГБОУ ВО УГАТУ (450008, г. Уфа, ул. К. Маркса, д. 12, e-mail: pgl.petrov@mail.ru).

Целищев Владимир Александрович (Уфа, Россия) - доктор технических наук, профессор, заведующий кафедрой «Прикладная гидромеханика» ФГБОУ ВО УГАТУ (450008, г. Уфа, ул. К. Маркса, д. 12, e-mail: pgl.ugatu@mail.ru).

Кудерко Дмитрий Александрович (Москва, Россия) - кандидат технических наук, директор центра проектирования АО «Технодинамика» (115184, г. Москва, ул. Большая Татарская, д. 35, стр. 5, e-mail: dm_kuderko@mail.ru).

\section{About the authors}

Pavel V. Petrov (Ufa, Russian Federation) - Csc in Technical Sciences, Associate Professor of Applied Hydromechanics Department, Ufa State Aviation Technical University (11, K. Marksa st., Ufa, 450008, Russian Federation; e-mail: pgl.petrov@mail.ru).

Vladimir A. Tselischev (Ufa, Russian Federation) - Doctor of Technical Sciences, Professor, Head of Department of Applied Hydromechanics, Ufa State Aviation Technical University (12, K. Marksa st., Ufa, 450008, Russian Federation; e-mail: pgl.ugatu@mail.ru).

Dmitry A. Kuderko (Moscow, Russian Federation) - Csc in Technical Sciences, Head of R@D center JSC "Technodinamika" (35, p. 5, Bolshaya Tatarskaya st., Moscow, 115184, Russian Federation; e-mail: dm_kuderko@mail.ru).

Получено 16.09.2021 